\title{
The Inclusion of Design Content in the Unit Operations Laboratory
}

\author{
Darin Ridgway, Valerie L. Young. Michael E. Prudich \\ Ohio University
}

\begin{abstract}
There has been an emphasis to include design throughout the chemical engineering curriculum. Open-ended projects with design content are commonly included in core courses. The unit operations lab offers another opportunity through the use and extension of experimental data. The range of student learning goals in the typical unit operations laboratory, including the planning and execution of experiments, the interpretation of experimental data, and gaining familiarity with process equipment, make a design task a natural extension. Examples of how these data can be utilized are (i) to determine if the equipment can accomplish a different task; (ii) to design a larger piece of equipment; or (iii) to develop a model which is then used for large-scale design.

At Ohio University we have instituted a design objective that each laboratory experiment is centered around. The design objective forces students to consider what data they need to collect, and analysis they need to employ. Examples for four experiments are presented here to demonstrate how the different aspects of design can be incorporated. Student response to this approach has been generally favorable.
\end{abstract}

Introduction

ABET defines design as follows, "Engineering design is the process of devising a system, component, or process to meet desired needs. It is a decision-making process (often iterative), in which the basic sciences and mathematics and engineering sciences are applied to convert resources optimally to meet a stated objective. ${ }^{1,}$

There has been an emphasis to include design throughout the chemical engineering curriculum. Open-ended projects with design content are commonly included in core courses. An aspect of design not as common, however, is the use and extension of experimental data from laboratory experiments. There is a range of student learning goals in the typical unit operations laboratory. These include the planning and execution of experiments, the interpretation of experimental data, and gaining familiarity with process equipment. These objectives make a design task a natural extension. Examples of how these data can be utilized are (i) to determine if the current lab equipment can be used for a different task; (ii) to design a largerscale piece of equipment; (iii) to develop a model which is then used for large-scale design. 
There have been examples in recent years of extending the traditional unit operations lab experience, some quite similar to that discussed below. Recently, McCallum and Estevez discussed a process design extension for a packed column experiment. ${ }^{2}$ In their experimental system, toluene is stripped from water using a column packed with 5/8" Pall rings. In the design extension trihalomethanes are to be stripped from water using the same packing material. Likewise, Davies and Langrish discussed the introduction of commercial relevance to the unit ops lab through two experiments; a filtration that required a process scale-up and a leaching experiment that required an economic optimization of an existing plant ${ }^{3}$.

Extensions of the traditional lab experience less similar to the approach presented here have also been presented. Marrero and Burkett discussed the reorganization of the unit ops lab course in order to put a feel of industrial practice on the course. This comprehensive approach included rewriting operating instructions and report format to match common industrial format and, more pertinent to this paper, a link of the experimental objective to a practical industrial problem $^{4}$. Myers used a simulated industrial-scale troubleshooting scenario as a method of directing the students' experimental procedure and reporting ${ }^{5}$. Middleberg discussed the inclusion of an exercise in the design of a laboratory experiment as a portion of the unit ops lab grade $^{6}$. Jayakumar et al. reported on a series of simulation modules that have been created for inclusion in laboratory courses. They offer the advantage of (i) overcoming size, complexity, and safety issues, (ii) including realistic time and budget constraints, and (iii) allowing increased emphasis on experimental design and data analysis ${ }^{7}$. A number of other modules have been created and reported on.

\section{Course Format}

At Ohio University the Unit Operations Laboratory is a two-quarter sequence taken during the senior year. The junior year thermodynamics, reactor design and unit operations courses are prerequisites, along with a course in experimental design. Students typically work in teams of three. Each experiment is led by a different faculty member. The instructor is responsible for all aspects of assigning of objectives, meeting with the students and grading the reports. The format includes an informal meeting with the instructor, a graded prelab in which the students are to present their experimental plan and expected data analysis, two weeks of lab time and a final written report.

The experiments are presented to the teams as a design objective. The specific experimental objectives are then developed to meet the design objective. The design objective forces students to consider what data they need to collect, and which analyses they need to employ. The team as a whole completes the experimental objectives, and these are what the written report specifically addresses. The design objective is completed and presented by an individual through a written technical memo and an oral presentation to the lab instructors. This has the added benefits of contributing a measure of individual content to the course grade and providing another forum for oral communication skills to be developed. Each student completes one design during the quarter. 


\section{Specific Examples}

What follows is a list of several of the projects used in the past year. They are selected to demonstrate the variety of design experiences, ranging from use of the current equipment for another task, design of larger-scale equipment to developing models for large-scale design. Included is a brief description of the experiment and an excerpt from the assignment that demonstrates the design problem assigned. There is some variation in the way that the questions are asked because of the course format in which each experiment is led by a different faculty member. The designs selected are intended to demonstrate the variety of responses received. No claim is made to what extent the individual designs mentioned were evaluated favorably by the faculty. The oral presentation offered an opportunity to offer feedback to the students as to the strengths and weaknesses of their design. There was no evidence that the students were merely reproducing others responses. If this is of concern it is fairly simple to change the design objective for each team.

\section{Fluid Flow Loop}

Apparatus and Experiment The apparatus consists of a series of pipes ranging from $1 / 2$ to 2 inch diameter, some in a vertical orientation. At various locations on the equipment are an orifice meter, vortex flow meter, globe, gate and ball valves, contractions, expansions, and elbows. Pressure taps are located throughout the system. Water is circulated from a holding tank using a 3/4 hp centrifugal pump.

Sample Design Problem “ “.. You have a 4000 gallon tank of methyl ethyl ketone at $150^{\circ} \mathrm{F}$. The fluid in the tank must be pumped to another tank located at a distance of $320 \mathrm{ft}$ horizintally and $50 \mathrm{ft}$ above the first tank. Discuss the possibility of using the pump in the lab for this job. Design a piping system to accomplish the task. Consider various pipe sizes and what this means for the expected performance."

Discussion The students were required to use their experimental results for the pump performance curve and for flow in a straight pipe, loss coefficients, etc. If their design used pipe diameters greater than 2", they were to use their experimental results as a basis for the design. The designs submitted ranged from $1 / 2$ " piping system requiring 32 hours to a 4 " system requiring 2.8 hours. The primary reasons for the range of designs were the large variation measured for the loss coefficients between the various teams, and the importance or attention the individual students gave to operating at the more efficient regions of their pump performance curve.

\section{Reaction Kinetics}

Apparatus and Experiment The equipment consists of a 4 liter stirred-tank reactor. It can be operated either as a CSTR or in batch mode. A second-order reaction between crystal violet dye (CVD) and sodium hydroxide is utilized. Samples are continuously withdrawn and plumbed 
through a diode-array spectrophotometer wired to a PC. The data consisting of CVD absorbances are displayed in real time and simultaneously recorded. Proper design of the procedure allows the kinetic parameters to be determined.

Sample Design Problem "Design a reactor to decolor 10,000 gallons per day of 0.0001 M CVD solution so that the discharge appears nearly clear. Specify reactor volume, residence time, temperature, concentration of $\mathrm{NaOH}$, and final concentration of CVD."

\section{$\underline{\text { Discussion }}$}

Most teams determine kinetic parameters by running the experiment in batch mode, then design a single CSTR to treat the waste stream. (The specified concentration of dye in the waste stream is outside the range of possible experimental conditions, preventing direct scaleup of an experimental run.) Reported reactor volumes ranged from 1200 to 5000 gallons; reported residence times from 2 to 10 hours. All used elevated temperature to enhance reaction rate; one design exceeded the boiling point of the reaction mixture. One design suggested accumulating the effluent and treating it in a batch reactor; another used three CSTRs in series. Thus far, no one has suggested a tubular flow reactor.

\section{Filtration}

Apparatus and Experiment The equipment consists of a plate-and-frame filter press. The frames are $1 \mathrm{ftx} 1 \mathrm{ft}$. The associated experimental objectives ask the team to develop models describing the filter medium resistance as a function of pressure drop and the specific cake resistance as a function of pressure drop and cake composition, considering the use of diatomaceous earth filter aid.

Sample Design Problem “... A mining operation being performed by your company produces a wastewater stream which has 4 wt \% limestone mixed into water. You are to evaluate the use of a plate-and-frame filter press to remove the limestone from the water. A filter aid (diatomaceous earth) can be added to the feed mixture to improve the operating characteristics. Your objective is to design a batch filter press system (with associated peripherals, piping, and valves) which will process $10 \mathrm{gpm}$ of a $4 \mathrm{wt} \%$ suspension of limestone. Your design must specify the size of the filter and the operating conditions for the filter. The costs of the filter aid, pump, and filter should be considered."

Discussion The students were to consider the downtime for the batch filtration process in meeting the 10 gpm objective. The designs presented had the following ranges for key variables from 6 to 15 plates, 1 to $4 \mathrm{ft}^{2}$ per plate, 10 to 200 psi pressure drop, 0.3 to $10 \mathrm{wt}$ percent diatomaceous earth, and a pump size from $0.4 \mathrm{hP}$ to $200 \mathrm{hP}$. The cycle times ranged from a 5 minute operating time with a 15 minute downtime to a 35 minute operating time with a 45 minute downtime. The wide variation in designs was primarily due to the difference in the models generated. The form of models required to meet the objectives were not specified and there was a large difference. 


\section{Membrane Separator}

Apparatus and Experiment The experimental equipment includes a bench-scale Permea membrane permeation unit that consists of four separation casings. The casings can be operated in any combination allowing co-current, counter-current, and mixed-flow operating regimes to be investigated. Feed gas (air) flow rate, as well as, feed gas and product gas pressures, can be controlled. The composition (percent $\mathrm{O}_{2}$ ) and flow rate of the product streams can be measured.

Sample Design Problem "How about that Global Warming?!? Boy, it's hot!!! Carbon dioxide $\left(\mathrm{CO}_{2}\right)$, a greenhouse gas, can be more economically removed from combustion off gases if it is present in higher than normal concentrations. Normal combustion practice calls for the use of atmospheric air to supply the oxygen $\left(\mathrm{O}_{2}\right)$ for combustion. Unfortunately, atmospheric air contains a boatload of nitrogen that ends up diluting the carbon dioxide in the combustion off gas. Your company, the Acme Permeation Station, is interested in applying its proprietary membrane permeation technology to this environmental problem. You are charged with designing a large pilot membrane permeation unit that will supply 1000 SCFM of $45 \%$ $\mathrm{O}_{2}$-enriched air to a natural gas combustion system that is currently operating at $10 \%$ excess air. In order to design the pilot unit you must be able to specify: (1) the operating pressure, (2) the inlet air flow rate, and (3) the area of membrane required in order to supply the required amount of $\mathrm{O}_{2}$-enriched air. Use your bench-scale membrane permeation unit in order to come up with an effective design.

In addition to your design, answer the following questions:

(1) What is the current concentration of $\mathrm{CO}_{2}$ (mole\%) in the combustion off gas from the natural gas combustion system? [Report both on wet and dry basis.] .

(2) What is the concentration of $\mathrm{CO}_{2}($ mole $\%)$ that will be expected in the combustion off gas once your membrane permeation system in installed? [Report both on wet and dry basis.].

(3) What is the expected composition of the "off gas" from the membrane permeation unit? What will you do with it? Why? “

Discussion Since the actual membrane area in the Permea unit is not known, the areas reported in the designs were reported as a scale of the lab equipment. These areas varied by more than an order of magnitude, from $2 \times 10^{5}$ to $1.2 \times 10^{6}$ area units. The reason for the difference depended on whether the students actually used the design equations to optimize the conditions or merely used a direct scale-up of an experimental condition that closely matched the design objective, a design method that they were specifically warned not to use.

\section{Student Response}

The students in Fall Quarter 2000 were surveyed about the assigned design problem (18 responses from 20 students). A seven point scale was utilized, ranging from "strongly agree" (7), through "neither agree or disagree" (4) to strongly disagree (1). Reported below are the 
overall averages \pm standard deviation for each of the question for all students and all experiments. Also reported are the high and low averages of the students from each specific experiment. Remember that each student completed an individual design from one of the experiments their group conducted.

\begin{tabular}{|l|l|l|l|l|}
\hline$\#$ & Question & $\begin{array}{l}\text { Overall } \\
\text { Average } \\
\pm \text { Std Dev }\end{array}$ & $\begin{array}{l}\text { High average } \\
\text { for any single } \\
\text { experiment }\end{array}$ & $\begin{array}{l}\text { Low average } \\
\text { for any single } \\
\text { experiment }\end{array}$ \\
\hline & $\begin{array}{l}\text { The UO lab design project was useful in focusing the } \\
\text { experimental plan we conducted. }\end{array}$ & $\begin{array}{l}4.94 \\
\pm 1.16\end{array}$ & 5.2 & 4.2 \\
\hline $\begin{array}{l}\text { The connection between the lab experiment and the } \\
\text { design problem was clear. }\end{array}$ & $\begin{array}{l}5.06 \\
\pm 1.30\end{array}$ & 5.8 & 4.2 \\
\hline $\begin{array}{l}\text { The UO lab design project was useful in connecting the } \\
\text { lab to the "real world". }\end{array}$ & $\begin{array}{l}4.94 \\
\pm 1.26\end{array}$ & 5.6 & 4.2 \\
\hline $\begin{array}{l}\text { I was adequately prepared to complete the assigned } \\
\text { design problem. }\end{array}$ & $\begin{array}{l}4.76 \\
\pm 1.71\end{array}$ & 6.0 & 3.5 \\
\hline & $\begin{array}{l}\text { The design problem should continue as a part of the } \\
\text { Unit Ops Lab course. }\end{array}$ & $\begin{array}{l}4.82 \\
\pm 1.55\end{array}$ & 5.6 & 4.4 \\
\hline
\end{tabular}

The scores indicate a generally favorable response to the inclusion of the design project in the Unit Operations lab course, based on the overall averages being above 4. It was interesting that the lowest overall average came from Question 4. This question caused the students to reflect most closely on their own ability and previous educational experience. Question 4 also showed both the highest and lowest individual experiment averages seen for any question. The score of 6.0 was from the Flow Loop experiment and the 3.5 score was from the Filter Press experiment. Of the four experiments in the Fall Quarter rotation the Flow Loop would be seen as the experiment that most closely was based upon previous course material, while the Filter Press required material that was not directly presented in any previous course.

\section{Conclusions}

A individual design project has been successfully included in the traditional Unit Operations Lab courses at Ohio University. A design problem is connected to each experiment, and one member of the group completes the design individually. The problems might ask the student to evaluate the ability of the lab equipment to complete a different task, to determine parameters which are then used to scale-up the equipment, or to create more general design equations used to complete a larger-scale design. The design problems were successful in focusing the experimental objectives for the students, connecting the laboratory to a "real-world" problem, and most importantly offering another opportunity to include a design component to the curriculum. 
Bibliography

1 “2000-2001 Criteria for Accrediting Engineering Programs - Conventional Criteria” p. 5.www.abet.org.

2 McCallum, C. L. and Estevez, A., 1999. "Introducing Process-Design Elements in the Unit Operations Lab," Chemical Engineering Education, Vol. 33 No 1, p. 66.

3 Davies, W.A. and Langrish, T.A.G., 1995, "Putting Commercial Relevance Into the Unit Operations Laboratory," Chemical Engineering Education, Vol. 29, No 1, p. 40.

4 Marrero, T.R., and Burkett, W.J., 1994, "Introducing Industrial Practice in the Unit Operations Lab," Chemical Engineering Education, Vol. 28, No 2, p. 128.

5 Myers, K.J., 1994, “Troubleshooting in the Unit Operations Laboratory,” Chemical Engineering Education, Vol. 28, No 2, p. 120.

6 Middleberg, A.P.J., 1995, "Laboratory Projects - Should Students Do Them or Design Them," Chemical Engineering Education, Vol. 29, No 1, p. 34.

7 Jayakumar, S., Squires, R.G., Reklaitis, G.V., and Grassi, K.S., 1995. "Simulating the Air Products Cryogenic Hydrogen Reactive Cooling Process," Chemical Engineering Education, Vol. 29, No 1, p. 26.

Biographical Information

Darin Ridgway is an Associate Professor of Chemical Engineering at Ohio University. He received his B.S. from RoseHulman Institute of Technology, M.S. from Louisiana Tech University and Ph.D. from Florida State University. His current research interest is in optimization of fungal fermentations.

Valerie L. Young is an Assistant Professor of Chemical Engineering at Ohio University. She received her B.S. from Lehigh University and Ph.D. from Virginia Tech University. Her current research interest is in quantification of trace organic species in the troposphere.

Michael E. Prudich is Professor and Chairman of the Chemical Engineering Department at Ohio University. His B.S., M.S., and Ph.D. degrees are from West Virginia University. He joined the faculty of Ohio University after working for Gulf Oil Company in Pittsburgh.

Proceedings of the 2001 American Society for Engineering Education Annual Conference \& Exposition

Copyright $\odot$ 2001, American Society for Engineering Education 